\title{
BMJ Open Study protocol: haemostatic efficacy and safety of preemptive antifibrinolysis with multidose intravenous tranexamic acid in elderly hip fracture patients: design of a prospective randomised controlled trial
}

Jiacheng Liu (D) , Yiting Lei, Junyi Liao, Xi Liang, Ning Hu, Wei Huang (D)

To cite: Liu J, Lei Y, Liao J, et al. Study protocol: haemostatic efficacy and safety of preemptive antifibrinolysis with multidose intravenous tranexamic acid in elderly hip fracture patients: design of a prospective randomised controlled trial. BMJ Open 2021;11:e047382. doi:10.1136/ bmjopen-2020-047382

- Prepublication history for this paper is available online. To view these files, please visit the journal online (http://dx.doi. org/10.1136/bmjopen-2020047382).

$\mathrm{JL}$ and $\mathrm{YL}$ are joint first authors.

Received 28 November 2020 Accepted 22 November 2021

Check for updates

(C) Author(s) (or their employer(s)) 2021. Re-use permitted under CC BY-NC. No commercial re-use. See rights and permissions. Published by BMJ.

Department of Orthopaedics, Orthopaedic Laboratory of Chongqing Medical University, The First Affiliated Hospital of Chongqing Medical University, Chongqing, People's Republic of China

Correspondence to

Professor Wei Huang;

huangwei68@263.net

\section{ABSTRACT}

Introduction Hip fracture surgery is often associated with substantial blood loss and a high allogeneic blood transfusion (ABT) rate. Preoperative hidden blood loss (HBL) has been observed clinically but there is little evidence for the efficacy of tranexamic acid (TXA) in controlling preoperative HBL. We designed a randomised controlled trial to evaluate the efficacy of preemptive antifibrinolysis with multidose intravenous TXA (IV-TXA) in reducing preoperative $\mathrm{HBL}$ in elderly patients with hip fractures.

Methods and analysis This is a prospective, randomised, placebo-controlled clinical trial. Patients older than 65 years diagnosed with primary unilateral femoral neck fracture or intertrochanteric fracture will be randomly assigned to group A (receiving $100 \mathrm{~mL}$ of intravenous normal saline every 12 hours preoperatively and $1.5 \mathrm{~g}$ of IV-TXA every 12 hours postoperatively for 3 days) or group $\mathrm{B}$ (receiving $1.5 \mathrm{~g}$ of IV-TXA every 12 hours preoperatively and $1.5 \mathrm{~g}$ of IV-TXA every 12 hours postoperatively for 3 days). The primary outcomes will be the hidden blood loss, haemoglobin decrease and ABT rate. The secondary outcomes include the levels of inflammatory factors (such as $C$ reactive protein) and coagulation and fibrinolysis parameters (such as D-dimer). Other outcomes such as injury time, length of stay and hospitalisation expenses will also be compared between groups.

Ethics and dissemination This study has been approved by the Ethics Committee of the First Affiliated Hospital of Chongqing Medical University. The findings of the study will be disseminated through peer-reviewed journals and conference presentations.

Trial registration number ChiCTR2100045960.

\section{INTRODUCTION}

Hip fracture is associated with complications such as chronic pain, disability, diminished quality of life and premature death. ${ }^{12}$ The reported mortality rate is as high as $30 \%$ in the first year after injury, and most patients with hip fractures require surgery. ${ }^{3}$ However,
Strengths and limitations of this study

- The randomised controlled trial (RCT) study design is the most robust methodology with which to assess the effectiveness of therapeutic interventions.

- This will be the first RCT to evaluate the haemostatic efficacy of multidose intravenous tranexamic acid (TXA) on preoperative hidden blood loss in elderly patients with hip fractures.

- The study protocol has been designed in strict accordance with the Consolidated Standards of Reporting Trials guidelines.

- The outcomes will be reported based on a 90-day follow-up period, which is theoretically sufficient for the safety assessment of TXA due to its short elimination half-life.

- This prospective RCT about preemptive antifibrinolysis will help to determine the optimal TXA regimen for geriatric patients with hip fractures.

the surgery sometimes induces severe blood loss that can be six times more than calculated during operation. ${ }^{4}$ As a result, $89.2 \%$ of patients are diagnosed with anaemia postoperatively. ${ }^{5}$ In clinical practice, anaemia is often treated with allogeneic blood transfusion (ABT). However, ABT is associated with transfusion reactions, immunosuppression, serious bacterial infection and increased hospital expenses. ${ }^{6}$ Therefore, researchers have explored several alternative therapies over the last few years, including antifibrinolysis.

As a representative antifibrinolytic agent, tranexamic acid (TXA) is a synthetic derivative of lysine that competitively blocks the lysine binding site on plasminogen to impede its activation, and thus inhibit the activity of the fibrinolysis system. ${ }^{7}$ TXA is effective 
in reducing blood loss in patients undergoing total hip arthroplasty and total knee arthroplasty. ${ }^{8}$ Furthermore, the same blood-sparing efficacy of TXA in patients with hip fracture has been demonstrated in several recent studies. $^{9-11}$

However, while the current TXA regimen for elderly patients with hip fractures is based on patients undergoing elective total joint arthroplasty (TJA), geriatric patients with hip fractures are quite a different population to patients undergoing elective TJA. First, the former experience hyperfibrinolysis twice (at the time of fracture and during surgery), while the latter experience hyperfibrinolysis only once (during surgery). This difference may contribute to the substantial preoperative hidden blood loss (HBL) in geriatric patients with hip fractures, and help explain why $44 \%$ of these patients have a lower than normal haemoglobin $(\mathrm{Hb})$ level before surgery. ${ }^{12}$ Second, despite the proven haemostatic efficacy of TXA administration intraoperatively and postoperatively, elderly patients with hip fractures still have a high blood loss volume. ${ }^{13}$ This may be because the preoperative HBL is reportedly $1473 \mathrm{~mL}$, and the widely used TXA regimen has little effects on the first hyperfibrinolysis event that occurs preoperatively. ${ }^{4}$ Furthermore, the $\mathrm{Hb}$ level is positively correlated with functional recovery, and anaemia is an independent risk factor for the inability to walk after the surgical treatment of hip fracture. ${ }^{14}{ }^{15} \mathrm{In}$ addition, osteoporotic fractures reportedly comprise a major constituent of fractures in China, mainly affecting persons aged 65 years or older. ${ }^{16}$ Due to multiple comorbidities and poor cardiopulmonary capacity, elderly patients with hip fractures appear to be frailer in the face of the substantial blood loss and systematic stress caused by the injury. Therefore, orthopedists first have to correct the underlying diseases owing to the poor preoperative body condition, which may delay the optimal operation timing. Hence, to decrease the risk of surgery and reduce the surgery delay in geriatric patients with hip fractures, a more positive haemostatic strategy should be implemented preoperatively.

Previous studies have focused on TXA administration in the intraoperative and postoperative periods, while there is a lack of evidence regarding the administration of multidose intravenous TXA (IV-TXA) preoperatively to reduce the preoperative HBL caused by the initial trauma. Therefore, we proposed the use of preemptive antifibrinolysis to maintain a continuous antifibrinolytic effect during the whole perioperative period via the administration of IV-TXA from immediately after admission. We hypothesised that this TXA regimen could decrease the preoperative HBL in elderly patients with hip fractures and lower the ABT rate.

\section{METHODS AND ANALYSIS}

\section{Aim}

Our main objective is to determine whether preemptive antifibrinolysis with multidose IV-TXA reduces blood loss
Table 1 Clinical classification of femoral neck fracture

\begin{tabular}{lll}
\hline Classification & Stage & Definition \\
\hline $\begin{array}{l}\text { Garden } \\
\text { Classification }\end{array}$ & Stage I & $\begin{array}{l}\text { Incomplete fracture (impact } \\
\text { valgus fracture). } \\
\text { Complete fracture without } \\
\text { displacement. }\end{array}$ \\
& Stage III & $\begin{array}{l}\text { Complete fracture with } \\
\text { partial displacement. } \\
\text { Complete fracture with full } \\
\text { displacement. }\end{array}$ \\
\hline
\end{tabular}

(especially preoperative HBL) in geriatric patients with hip fractures and to assess the safety of this regimen.

\section{Study design}

This is a prospective, double-blinded randomised controlled trial (RCT). Patients aged 65 years or older who are diagnosed with femoral neck fracture or intertrochanteric fracture on plain radiography or CT will be assessed for eligibility. We will further divide the two types of fractures into different subgroups according to the widely accepted classification methods (tables 1 and 2). ${ }^{1718}$ All patients must provide informed consent before enrolment. The flow chart and schedule of this trial are shown in figure 1 and table 3 . This study has been approved by the Ethics Committee of the First Affiliated Hospital of Chongqing Medical University (approval number: 202046-2). This clinical trial is planned to start on June 2021 in the orthopaedic ward of the First Affiliated Hospital of Chongqing Medical University (Chongqing, China) and is estimated to end by May 2022. There will be 11 investigators, including 3 senior orthopaedic surgeons $(\mathrm{WH}$, $\mathrm{XL}$ and $\mathrm{NH}$ ) with more than 20 years of clinical experience, 6 orthopaedic physicians (HC, CC, QC, CZ, KL and $\mathrm{JL}$ ), and 2 data collectors who are also statisticians (JL and YL).

\section{Inclusion and exclusion criteria}

The study inclusion and exclusion criteria are outlined in table 4 .

\begin{tabular}{|c|c|c|}
\hline Classification & Stage & Definition \\
\hline \multirow[t]{5}{*}{$\begin{array}{l}\text { Evans-Jensen } \\
\text { Classification }\end{array}$} & Stage I A & $\begin{array}{l}\text { Simple two-part fracture } \\
\text { without displacement. }\end{array}$ \\
\hline & Stage I B & $\begin{array}{l}\text { Simple two-part fracture with } \\
\text { displacement. }\end{array}$ \\
\hline & Stage II A & $\begin{array}{l}\text { Three-part fracture involving } \\
\text { the greater trochanter. }\end{array}$ \\
\hline & Stage II B & $\begin{array}{l}\text { Three-part fracture involving } \\
\text { the lesser trochanter. }\end{array}$ \\
\hline & Stage III & $\begin{array}{l}\text { Four-part fracture involving } \\
\text { both the greater and lesser } \\
\text { trochanter. }\end{array}$ \\
\hline
\end{tabular}




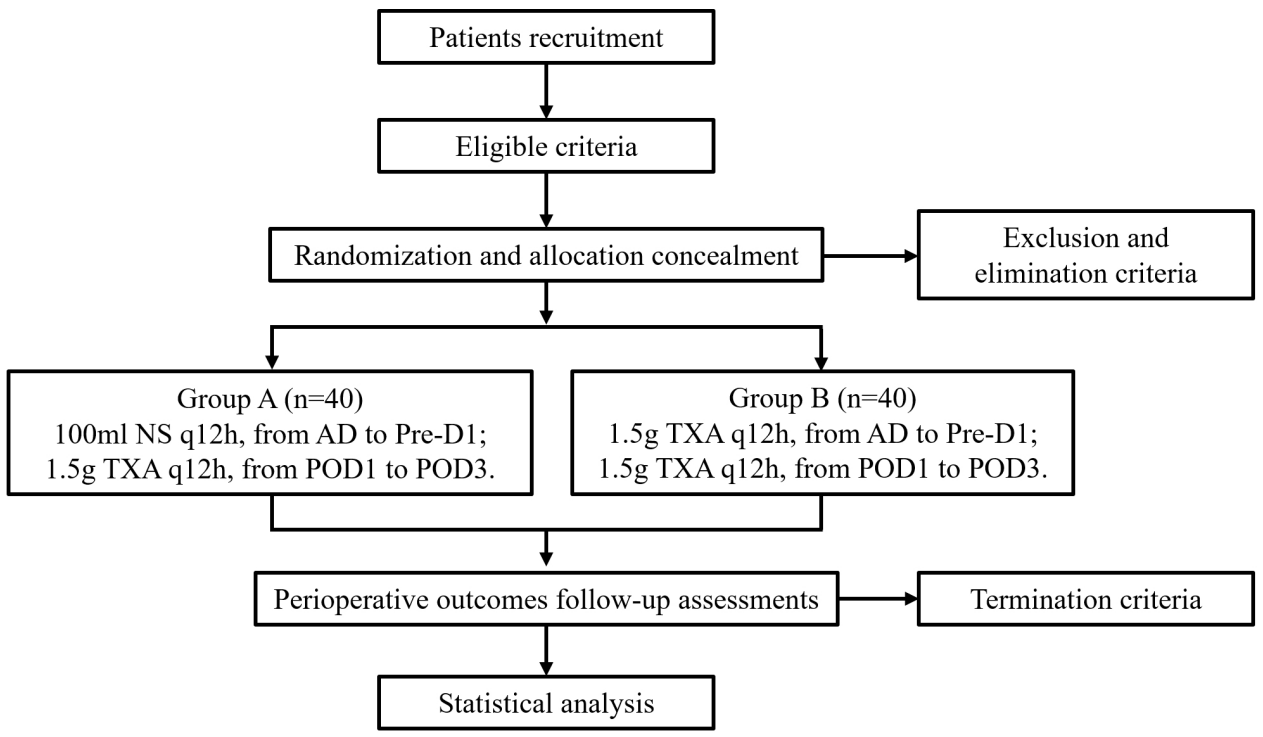

Figure 1 The study flow chart. AD, admission day; NS, normal saline; POD1, postoperative day 1; POD3, postoperative day 3; Pre-D1, 1 day before operation; TXA, tranexamic acid.

\section{Elimination criteria}

1. Patients who the researchers believe will benefit from withdrawal from the study.

2. Patients who decide to withdraw from the study.

\section{Termination criteria}

Patients will be terminated once any one of the following events occurs:

1. Shock.

2. Allergic symptoms.

3. Reactive dermatitis, hypotension, dizziness, headache, vertigo, convulsions, or blurred vision.

4. Intracranial thrombosis and/or haemorrhage.

5. Deep vein thrombosis (DVT) or pulmonary embolism (PE).

\section{Participants and informed consent}

This trial will only include elderly patients with hip fractures who meet all the above-mentioned criteria and provide written informed consent. Bone mineral density (BMD) assessment by dual-energy X-ray absorptiometry will be required for all elderly hip fracture patients in our centre. Intertrochanteric fracture patients with osteoporosis (defined as BMD $\leq-2.5$ ) will receive hip arthroplasty due to the high prevalence of unsatisfactory functional results of internal fixation, ${ }^{19}{ }^{20}$ while those without osteoporosis will receive internal fixation. The medial fragment will be routinely retained with cerclage wire during arthroplasty using a standard femoral stem among the intertrochanteric fractures. Additionally, femoral neck fracture patients classified as Garden III/IV will be treated with hip arthroplasty, while those classified as Garden I/II will receive internal fixation. Only those scheduled for hip arthroplasty will be assessed for the enrolment of this trial.

Before study participation, patients will receive a printed copy of the benefits, discomforts and potential risks they may receive from participation in this trial. Patients are free to discuss the benefits and risks of the trial with their relatives, friends or private doctors to help them decide whether to participate. All patients will be informed that they can withdraw from the trial at any time. Patients will receive compensation for any losses if they experience related injury during the study. No personal information of the enrolled patients will be revealed.

\section{Sample size calculation}

Sample size calculation was based on the data from a previous study of HBL in patients with intertrochanteric fractures, which showed that the HBL volumes in the TXA and control groups were $410.42 \pm 178.23 \mathrm{~mL}$ and $571.19 \pm 218.13 \mathrm{~mL}$, respectively. ${ }^{11}$ Considering a potential dropout rate of $15 \%$, the trial requires 80 patients to yield a power of $90 \%$ with a significance level of 0.05 . All sample size calculations were performed using PASS 2011 software (NCSS, LLC, Kaysville, Utah, USA).

\section{Randomisation and allocation concealment}

Enrolled patients will be randomly assigned into two groups in a 1:1 ratio. Randomisation will be done by SPSS V.24.0 software (IBM Corporation) in blocks of 2, 4 and 8 , and stratified by fracture type (femoral neck fracture or intertrochanteric fracture) and injury time ( $\leq 72$ hours or $>72$ hours). Injury time is defined as the time from fracture occurrence to the first administration of TXA or normal saline. This cut-off is set based on the 72-hour ongoing bleeding state after injury in the elderly hip fracture patients. ${ }^{21}$ Under the local hierarchical medical system, hip fracture patients are usually referred to our centre (a tertiary A hospital), and thus the injury time could vary from hours to days. The group data will be collected by two statisticians and placed into opaque envelopes and entered into a computer using encryption. Only the two statisticians are authorised to 
Table 3 The schedule of trial enrolment and assessments

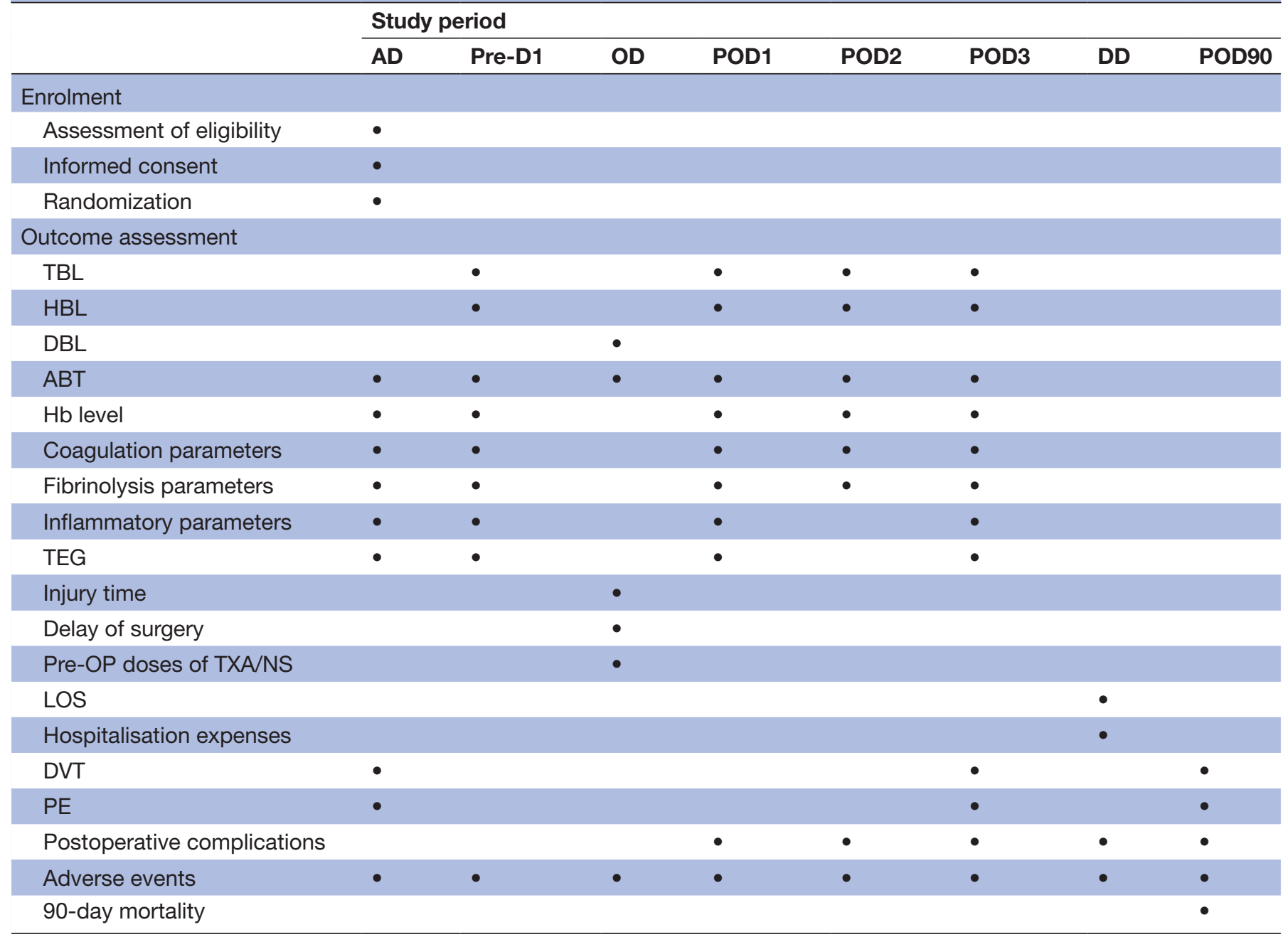

ABT, allogeneic blood transfusion; AD, admission day; DBL, dominant blood loss; DD, discharge day; DVT, deep vein thrombosis; Hb, haemoglobin; HBL, hidden blood loss; LOS, length of stay; NS, normal saline; OD, operative day; PE, pulmonary embolism; POD1, postoperative day 1; POD2, postoperative day 2; POD3, postoperative day 3; POD90, postoperative day 90; Pre-D1, 1 day before operation; Pre-OP, preoperative; TBL, total blood loss; TEG, thromboelastography; TXA, tranexamic acid.

check the group allocations and apply the corresponding treatment.

\section{Blinding}

Depending on their group allocation, patients will receive either $1.5 \mathrm{~g}$ of TXA or $100 \mathrm{~mL}$ of NS preoperatively. The TXA will be diluted in $100 \mathrm{~mL}$ NS so that the packaging of the two different injections is identical. All patients and clinicians will remain blinded until the end of the data analysis.

\section{Surgery and anaesthesia}

All patients will receive hemi- or total hip arthroplasty under general anaesthesia performed by three senior surgeons (WH, XL and $\mathrm{NH}$ ). To reduce the dosage and adverse effects of the anaesthetic agents, we routinely use intravenous inhalational anaesthesia. Anaesthesia will be induced using propofol, midazolam, sufentanil, and vecuronium, and maintained using sevoflurane and remifentanil. The specific doses of the anaesthetic agents for each patient will be calculated based on the patient's weight. As intraoperative antifibrinolytic treatment, both groups will receive an intravenous injection of $1.5 \mathrm{~g}$ of TXA $30 \mathrm{~min}$ before surgery, and a local injection of $1.0 \mathrm{~g}$ of TXA into the femoral medullary cavity during surgery. After the implantation of the prostheses, we will reset the joint and assess the stability and function. Before closure, C-arm fluoroscopy will be conducted to check the position of the implanted prostheses. After surgery, we routinely use elastic bandage to bind the affected limb to reduce postoperative swelling, and patients are required to wear T-shoes to prevent joint dislocation. No drainage tube will be placed in routine hip arthroplasty.

\section{Intraoperative blood loss}

As no drainage tube will be placed, the intraoperative blood loss will be calculated using the following formula: intraoperative blood loss=the volume of fluid in the negative pressure drain - the volume of NS used. 
Table 4 Inclusion and exclusion criteria

\begin{tabular}{ll}
\hline Inclusion criteria & Exclusion criteria \\
\hline 1) Over 65 years old; & $\begin{array}{l}\text { 1) Complicated with multiple } \\
\text { fractures or open fractures; }\end{array}$ \\
$\begin{array}{ll}\text { 2) Diagnosed with a primary, } \\
\text { unilateral, recent hip fracture } \\
\text { (femoral neck fracture of } \\
\text { intertrochanteric fracture) by X- } \\
\text { ray or CT scan; }\end{array}$ & $\begin{array}{l}\text { 2) Active bleeding (like } \\
\text { gastrointestinal bleeding, cerebral } \\
\text { haemorrhage, etc); }\end{array}$ \\
$\begin{array}{ll}\text { 3) Receiving hemi- or total hip } \\
\text { arthroplasty. }\end{array}$ & $\begin{array}{l}\text { 3) Systematic thromboembolism } \\
\text { (DVT, PE, etc); }\end{array}$ \\
& 4) Coagulation dysfunction; \\
& $\begin{array}{l}\text { 5) Combined with serious } \\
\text { neuromuscular diseases; }\end{array}$ \\
& $\begin{array}{l}\text { 6) Allergic to TXA. } \\
\text { 7) Patients who are dementially } \\
\text { reduced or with cognitive } \\
\text { dysfunction that cannot give } \\
\text { informed consent, or decline to } \\
\text { participate. }\end{array}$ \\
\hline
\end{tabular}

$\mathrm{CT}$, computed tomography; DVT, deep vein thrombosis; PE, pulmonary embolism; TXA, tranexamic acid.

\section{Study interventions}

After admission, both groups will be treated with skin traction therapy for immobilisation; the traction weight will be less than $5 \mathrm{~kg}$, and will be determined based on the weight of the patient. To minimise gastrointestinal adverse effects, selective cyclooxygenase-2 (COX-2) inhibitors such as celecoxib and etoricoxib will be used for preoperative analgesia. The haematocrit value and $\mathrm{Hb}$ level will be recorded daily during the perioperative period. In accordance with our hospital protocol, the indication for ABT will be a Hb level of $<70 \mathrm{~g} / \mathrm{L}$, or the onset of symptomatic anaemia (dizziness, fatigue, palpitation and paleness) with an $\mathrm{Hb}$ level of $70-100 \mathrm{~g} / \mathrm{L}$. The specific interventions in the two groups are listed below.

1. In group A, patients will be administered $100 \mathrm{~mL}$ of NS intravenously every 12 hours from the day of admission $(\mathrm{AD})$ to the day before surgery (Pre-D1). From postoperative day (POD) 1 to POD3, patients will be administered $1.5 \mathrm{~g}$ of TXA $+100 \mathrm{~mL}$ of NS intravenously every 12 hours.

2. In group B, patients will be administered $1.5 \mathrm{~g}$ of TXA $+100 \mathrm{~mL}$ of NS intravenously every 12 hours from the AD to the Pre-D1. The same dose of TXA will be injected intravenously every 12 hours from POD1 to POD3.

3. On the day of surgery, in addition to a local administration of $1.0 \mathrm{~g}$ of TXA, both groups will receive $1.5 \mathrm{~g}$ of IV-TXA $30 \mathrm{~min}$ before surgery and again 12 hours later. The use of TXA is based on the standard of the China Food and Drug Administration (YBH22332005). TXA is produced by Guangzhou Baiyunshan Tianxin Pharmaceutical Co. (approval number: National Drug Standard H20056985).

\section{Pain management and rehabilitation}

All patients will receive an ultrasound-guided femoral nerve block immediately after the closure of the incision as postoperative analgesia. In addition, a patientcontrolled analgesia pump combined with intravenous administration of a selective COX-2 inhibitory agent (such as parecoxib sodium) will be used for the first 3 days after surgery. From POD4, the intravenous analgesics will be discontinued and analgesia will comprise oral selective COX-2 inhibitors, such as celecoxib and etoricoxib. All patients will be encouraged to start rehabilitation exercises from POD1. Professional rehabilitation physicians will provide one-on-one rehabilitation treatment to each patient postoperatively. Walking aids will be used to speed the functional recovery postoperatively and shorten the hospital length of stay.

\section{Antibiotic therapy}

As perioperative prophylactic antibiotic therapy, $1.5 \mathrm{~g}$ of cefuroxime sodium will be administered $30 \mathrm{~min}$ before the surgery and repeated every 12 hours from POD1 to POD3.

\section{Prevention and monitoring of thrombotic events}

Low-molecular-weight-heparin sodium (4000 IU one time a day) will be injected subcutaneously to prevent thrombotic events (such as DVT and PE) for 24 hours after surgery. After discharge, patients will be instructed to take rivaroxaban $(10 \mathrm{mg}$ one time a day) until POD35. Patients will be encouraged to do as much rehabilitation exercise as they can to enhance the physical prevention of DVT. All enrolled patients will receive colour Doppler ultrasonography of the veins in both lower extremities on POD3 and POD90 to check for short-term DVT. In addition, computed tomographic pulmonary angiography (CTPA) will be conducted if any onset symptoms or signs of PE develop (eg, cyanosis, haemoptysis, thoracalgia, or dyspnoea).

\section{Outcomes}

Primary outcomes

$H B L, H b$ decrease and $A B T$

Our method to calculate HBL is based on the two formulas reported by Nadler and Gross. ${ }^{22} 23$ The specific calculations are as follows:

1. Patient blood volume $(\mathrm{PBV})=\mathrm{k} 1 \times$ height $^{3}(\mathrm{~m})+\mathrm{k} 2 \times-$ weight $(\mathrm{kg})+\mathrm{k} 3$ (For male: $\mathrm{k} 1=0.3669$, $\mathrm{k} 2=0.03219$, $\mathrm{k} 3=0.6041$. For female: $\mathrm{k} 1=0.3561, \mathrm{k} 2=0.03308$, $\mathrm{k} 3=0.1833$ ).

2. Total red blood cell volume loss=PBV $\times\left(\mathrm{Hct}_{0}-\mathrm{Hct}_{\mathrm{n}}\right)$, where $\mathrm{Hct}_{0}$ is the haematocrit on the admission day and Hct ${ }_{n}$ is the haematocrit on the nth day after admission.

3. Theoretical blood loss $\left(\mathrm{T}_{\text {heo }} \mathrm{BL}\right)=$ total red blood cell volume loss / Hct $_{0}$.

4. Perioperative blood loss $(\mathrm{PBL})=$ dominant blood loss (DBL) $+\mathrm{HBL}=\mathrm{T}_{\text {heo }} \mathrm{BL}+$ blood transfusion volume (BTV). 
5. $\mathrm{HBL}=\mathrm{T}_{\text {heo }} \mathrm{BL}+\mathrm{BTV}-\mathrm{DBL}$.

On the AD, Pre-D1, POD1, POD2, and POD3, the HBL will be calculated based on the haematocrit value, and the $\mathrm{Hb}$ levels will be recorded. As no drainage tube is placed in our routine hip arthroplasty, intraoperative blood loss will be recorded as DBL in this study. In addition, the frequency and volume of $\mathrm{ABT}$ will also be monitored.

\section{Secondary outcomes}

\section{Inflammatory markers}

The levels of erythrocyte sedimentation rate, $\mathrm{C}$ reactive protein, procalcitonin, interleukin (IL) 6, IL-8, IL-10 and tumour necrosis factor $\alpha$ will be recorded on the AD, PreD1, POD1 and POD3.

\section{Coagulation and fibrinolysis variables}

Coagulation and fibrinolysis variables, including prothrombin time, prothrombin time ratio, international normalised ratio, prothrombin activity, activated partial thromboplastin time, thrombin time, fibrinogen, fibrinogen degradation product and D-dimer (D-D) will be tested on the AD, Pre-D1, POD1, POD2 and POD3. Coagulation factors (II, V, VII, VIII, IX, X, XI and XII) will be recorded on the AD, Pre-D1, POD1 and POD3.

\section{Thromboelastography}

To monitor the overall dynamic changes in blood coagulation, we will assess the TEG variables (clot time, period to $2 \mathrm{~mm}$ amplitude (R); clot time, period from 2 to $20 \mathrm{~mm}$ amplitude (K); alpha angle (slope between $\mathrm{R}$ and $\mathrm{K}$ ); maximum strength (MA); lysis within $30 \mathrm{~min}$ after MA (LY30); estimate per cent lysis within $30 \mathrm{~min}$ after MA (EPL); comprehensive coagulation index) on the $\mathrm{AD}$, Pre-D1, POD1 and POD3.

All laboratory tests will be performed in the Department of Clinical Laboratory of the First Affiliated Hospital of Chongqing Medical University, and all variables will be assessed by investigators not involved in this trial.

\section{Other outcomes and follow-up}

Other recorded outcomes will include the injury time, surgery delay (time from admittance to surgery) and preoperative doses of TXA and NS. Wound complications (surgical site exudation, bleeding, haematoma, superficial or deep infection) and other postoperative complications (eg, pulmonary infection and urinary tract infection) will be monitored until POD90. Patients will be followed up by telephone on POD90 to identify postoperative complications, DVT, PE and other adverse events. In addition, the 90-day postoperative mortality data will be obtained by telephone follow-up and recorded in the case report forms (CRFs).

\section{Adverse events}

1. DVT diagnosed by Doppler ultrasonography, with or without symptoms (such as acute onset, affected limb swelling, severe pain, or marked tenderness at the femoral triangle or/and leg).
2. PE diagnosed by doppler ultrasonography, CTPA, or venography, with or without clinical manifestations (such as cough, chest tightness, palpitations, haemoptysis, shortness of breath, dizziness, shock, cyanosis, increased respiratory rate, or arteriovenous filling or pulsation).

3. Substantial swelling or generalised shallow venous tension of the affected limb.

All patients will be monitored continuously for adverse events from the $\mathrm{AD}$ to POD90.

\section{Adverse event treatment}

Adverse events will be classified in accordance with the five-level scoring system of the Common Terminology Criteria for Adverse Events and recorded in the CRFs. In addition, the associations between adverse events and drug use during hospitalisation and follow-up will be evaluated.

Serious adverse events are defined as events that cause defects, teratogenicity, permanent damage to organ function, permanent or substantial disability, prolonged length of stay, cancer, or death. If serious adverse events occur, the researcher will immediately initiate appropriate treatment and report it to the hospital and ethics committee.

\section{Trial end}

This trial will be completed after the collection of the data from the final follow-up assessment of the last enrolled patient.

\section{Data management}

The CRF data will be entered into a computer by two independent trained research assistants and will be periodically checked by the hospital's independent investigators.

\section{Statistical analysis}

After data collection, we will conduct the following analyses using SPSS V.24.0 software (IBM Corporation,).

1. Descriptive analyses will comprise the means (with SD) for continuous variables, and the frequencies (with percentages) for categorical or discrete variables.

2. For continuous variables like HBL, an independent ttest will be performed to evaluate the efficacy of TXA in reducing blood loss.

3. Qualitative variables (such as the frequency of transfusion) will be analysed using the $\chi^{2}$ test.

\section{Patient and public involvement}

Patients and this public were not involved in the design of this protocol.

\section{Ethics and dissemination}

This study has been approved by the ethics committee of the First Affiliated Hospital of Chongqing Medical University (ethical approval number: 2020-46-2). We will collect and use the patients' data with permission. The data will be available from the corresponding author on 
reasonable request. All data will be processed under the rules of the Chinese government and relevant laws.

\section{DISCUSSION}

Although numerous studies have consistently proved the positive blood-sparing effect of TXA, the optimal TXA regimen for patients with hip fractures remains controversial. High-quality evidence for the potential haemostatic effectiveness of TXA in reducing the preoperative HBL induced by the initial fracture is scarce. The main goal of this RCT is to provide level I evidence for the development of guidelines for TXA administration in elderly patients with hip fractures.

Geriatric patients with hip fractures represent a quite different population from patients undergoing elective TJA, as patients with hip fractures experience hyperfibrinolysis twice (at the time of the initial injury and during the subsequent surgery). The fibrinolysis system is activated after the initial trauma and continuous HBL is occurring before surgery. ${ }^{10}{ }^{24}$ This is in accord with our previous published work and may suggest an ongoing hyperfibrinolysis from admission to POD $3 .{ }^{25}$ However, to date, studies have only reported the haemostatic efficacy of TXA on the blood loss related to surgery, while there is a lack of evidence about the blood-sparing efficacy of TXA on the preoperative HBL caused by the initial fracture. Considering that fibrinolysis is a cascade reaction and is best inhibited in the initial period, we hypothesised that implementing TXA immediately after admission and maintaining a continuous antifibrinolytic effect during the whole perioperative period would provide further blood conservation in geriatric patients with hip fractures. In addition, according to previous pharmacological studies, the administration of $15 \mathrm{mg} / \mathrm{kg}$ of IV-TXA two times a day effectively reduces blood loss. ${ }^{26}{ }^{27}$ Considering that few older adults in China weigh over $100 \mathrm{~kg}$, we decided to use a dose of $1.5 \mathrm{~g}$ of IV-TXA every 12 hours. This regimen also meets the daily required dose of TXA (3-6g) for bleeding patients. ${ }^{26}$

According to a Chinese national survey performed in 2014, the most common injury mechanism for hip fractures is low energy injuries (like slips and falls), and the proportion of patients with hip fractures aged 65 years or older is $10.1 \% .^{16}$ Elderly patients with hip fractures often have multiple comorbidities such as congestive heart failure, chronic pulmonary disease and diabetes, ${ }^{28}$ and are thus more susceptible to adverse events from blood loss and immobilisation owing to poor cardiopulmonary function. In addition, due to the obvious blood loss and systematic stress after injury, elderly patients with hip fractures may fail to receive timely surgical treatment due to severe anaemia and degradation of underlying diseases. Therefore, these vulnerable patients require an effective and timely blood-sparing intervention. The PBL of geriatric patients with hip fractures consists of two parts: preoperative HBL induced by the initial fracture and haemorrhage caused by surgery. However, current haemostatic management only focuses on the intraoperative and postoperative periods, which may be less appropriate for elderly patients with hip fractures who have already experienced substantial HBL before surgery. ${ }^{24}$ Hence, we believe that intervention to minimise preoperative HBL is the key to further decrease blood loss, and designed this RCT to evaluate the potential haemostatic effect of preemptive antifibrinolysis with multidose IV-TXA in elderly patients with hip fractures.

In this trial, both groups will be treated with TXA intraoperatively and postoperatively to minimise the blood loss related to surgery. In addition, each group will receive a different preoperative intervention (either IV-TXA or an equal amount of NS) to evaluate the efficacy of preemptive antifibrinolysis. If our results confirm the haemostatic efficacy of preoperative TXA, this may change the current TXA regimen for geriatric patients with hip fractures and provide a novel strategy to reduce the preoperative HBL, hasten the initiation of surgery after injury, lower the surgical risks and improve the outcome.

Acknowledgements The authors gratefully acknowledge the contributions of the clinicians and nurses working at the orthopaedic ward of the First Affiliated Hospital of Chongqing Medical University for their efforts to prepare and undertake this study. We thank Kelly Zammit, BVSc, from Liwen Bianji (Edanz) (www.liwenbianji. $\mathrm{cn} / \mathrm{ac}$ ) for editing the English text of a draft of this manuscript. Jiacheng Liu wants to thank Jianying Zhong and Bin Liu particularly for the great love and care over the years.

Contributors $\mathrm{XL}, \mathrm{NH}$ and JuL designed the study while JiL and YL conceived the study. The study protocol was drafted by WH. All authors have read and approved the final manuscript of this study protocol. WH is the study guarantor.

Funding The authors have not declared a specific grant for this research from any funding agency in the public, commercial or not-for-profit sectors.

Competing interests None declared.

Patient and public involvement Patients and/or the public were not involved in the design, or conduct, or reporting, or dissemination plans of this research.

Patient consent for publication Not applicable.

Provenance and peer review Not commissioned; externally peer reviewed.

Open access This is an open access article distributed in accordance with the Creative Commons Attribution Non Commercial (CC BY-NC 4.0) license, which permits others to distribute, remix, adapt, build upon this work non-commercially, and license their derivative works on different terms, provided the original work is properly cited, appropriate credit is given, any changes made indicated, and the use is non-commercial. See: http://creativecommons.org/licenses/by-nc/4.0/.

ORCID iDs

Jiacheng Liu http://orcid.org/0000-0001-5115-8682

Wei Huang http://orcid.org/0000-0001-9116-2985

\section{REFERENCES}

1 Zuckerman JD. Hip fracture. N Engl J Med 1996;334:1519-25.

2 Dhanwal DK, Dennison EM, Harvey NC, et al. Epidemiology of hip fracture: worldwide geographic variation. Indian J Orthop 2011;45:15-22.

3 Moran CG, Wenn RT, Sikand M, et al. Early mortality after hip fracture: is delay before surgery important? J Bone Joint Surg Am 2005;87:483-9.

4 Foss NB, Kehlet $\mathrm{H}$. Hidden blood loss after surgery for hip fracture. $J$ Bone Joint Surg Br 2006;88:1053-9.

5 Wu X-D, Liu J-C, Li Y-J, et al. The necessity of routine postoperative laboratory tests after total hip arthroplasty for hip fracture in a semiurgent clinical setting. J Orthop Traumatol 2020;21:19.

6 Vamvakas EC, Blajchman MA. Transfusion-related mortality: the ongoing risks of allogeneic blood transfusion and the available strategies for their prevention. Blood 2009;113:3406-17. 
7 Virani SR, Dahapute AA, Panda I, et al. Role of local infiltration of tranexamic acid in reducing blood loss in peritrochanteric fracture surgery in the elderly population. Malays Orthop J 2016;10:26-30.

8 Duncan CM, Gillette BP, Jacob AK, et al. Venous thromboembolism and mortality associated with tranexamic acid use during total hip and knee arthroplasty. J Arthroplasty 2015;30:272-6.

9 Lei J, Zhang B, Cong Y, et al. Tranexamic acid reduces hidden blood loss in the treatment of intertrochanteric fractures with PFNA: a single-center randomized controlled trial. J Orthop Surg Res 2017;12:124.

10 Wang W, Yu J. Tranexamic acid reduces blood loss in intertrochanteric fractures: a meta-analysis from randomized controlled trials. Medicine 2017;96:e9396.

11 Zhou X-D, Zhang Y, Jiang L-F, et al. Efficacy and safety of tranexamic acid in Intertrochanteric fractures: a single-blind randomized controlled trial. Orthop Surg 2019;11:635-42.

12 Spahn DR. Anemia and patient blood management in hip and knee surgery: a systematic review of the literature. Anesthesiology 2010;113:482-95.

13 Tengberg PT, Foss NB, Palm H, et al. Tranexamic acid reduces blood loss in patients with extracapsular fractures of the hip: results of a randomised controlled trial. Bone Joint J 2016;98-B:747-53.

14 Lawrence VA, Silverstein JH, Cornell JE, et al. Higher Hb level is associated with better early functional recovery after hip fracture repair. Transfusion 2003;43:1717-22.

15 Foss NB, Kristensen MT, Kehlet H. Anaemia impedes functional mobility after hip fracture surgery. Age Ageing 2008;37:173-8.

16 Chen W, Lv H, Liu S, et al. National incidence of traumatic fractures in China: a retrospective survey of 512187 individuals. Lancet Glob Health 2017;5:e807-17.

17 Jensen JS, Michaelsen M. Trochanteric femoral fractures treated with McLaughlin osteosynthesis. Acta Orthop Scand 1975;46:795-803.
18 Garden RS. Stability and Union in subcapital fractures of the femur. $J$ Bone Joint Surg Br 1964;46:630-47.

19 Ju J-B, Zhang P-X, Jiang B-G. Hip replacement as alternative to intramedullary nail in elderly patients with unstable Intertrochanteric fracture: a systematic review and meta-analysis. Orthop Surg 2019;11:745-54.

20 Kim S-Y, Kim Y-G, Hwang J-K. Cementless calcar-replacement hemiarthroplasty compared with intramedullary fixation of unstable intertrochanteric fractures. A prospective, randomized study. J Bone Joint Surg Am 2005;87:2186-92.

21 Tian S, Li H, Liu M, et al. Dynamic analysis of perioperative hidden blood loss in Intertrochanteric fractures. Clin Appl Thromb Hemost 2019;25:107602961882327.

22 Gross JB. Estimating allowable blood loss: corrected for dilution. Anesthesiology 1983;58:277-80.

23 Nadler SB, Hidalgo JH, Bloch T. Prediction of blood volume in normal human adults. Surgery 1962;51:224-32.

24 Smith GH, Tsang J, Molyneux SG, et al. The hidden blood loss after hip fracture. Injury 2011;42:133-5.

25 Wu X-D, Tian M, He Y, et al. Efficacy of a three-day prolonged-course of multiple-dose versus a single-dose of tranexamic acid in total hip and knee arthroplasty. Ann Transl Med 2020;8:307.

26 Verstraete M. Clinical application of inhibitors of fibrinolysis. Drugs 1985;29:236-61.

27 Rö JS, Knutrud O, Stormorken H. Antifibrinolytic treatment with tranexamic acid (AMCA) in pediatric urinary tract surgery. $J$ Pediatr Surg 1970;5:315-20.

28 Brauer CA, Coca-Perraillon M, Cutler DM, et al. Incidence and mortality of hip fractures in the United States. JAMA 2009;302:1573-9. 